\title{
MANEJO DA BATATA-DOCE NO CONTROLE DA BROCA- DA-RAIZ, A NÍVEL DO PRODUTOR
}

\section{Marcelo Bezerra de Andrade ${ }^{1}$ Antônio Fernando de Souza Leão Veiga ${ }^{2}$ RESUMO}

O trabalho foi conduzido em propriedade particular da Fazenda Rio Gramame, município de Pedras de Fogo, Paraíba, em solo francoareno-argiloso, plano e em sistema de sequeiro (com chuvas). Objetivouse avaliar a eficácia do sistema de manejo do cultivo da batata-doce (MCBd) quando comparado com o do agricultor (SCAg), no controle dos danos e perdas (deságio comercial) causados pela broca-da-raiz, Euscepes postfasciatus (Fairmaire), em dois períodos de colheita (4 e 5 meses), a nível de produtor. Usaram-se seis blocos casualizados com 5 tratamentos e colheitas aos 4 e 5 meses do plantio. Testou-se o sistema de manejo do cultivo de batata-doce (MCBd), com as seguintes técnicas de cultivo: imersão das ramas de plantio em calda inseticida à base de Clorpirifós (Lorsban $45 \mathrm{CE}$ ) a $0,1 \%$ do produto comercial por 20 minutos; uso de plantio de pontas de ramas e técnica do "laço" (dobra da base) a $15 \mathrm{~cm}$ de profundidade, no sistema mecanizado de leirão, com adubação química. Adicionaram-se ao MCBd, os tratamentos de cobertura morta com palha de cana-de-açúcar (PSCA) e adubação orgânica com esterco de gado (AOEG). Comparou-se com o sistema convencional do agricultor ( $\mathrm{SCAg}$ ). Todas as ramas de plantio foram oriundas de áreas infestadas pela broca. Mediram-se os danos $(\% \mathrm{~d})$ nas raízes tuberosas, produtividade $(\mathrm{t} / \mathrm{ha})$ e calcularam-se as perdas (coeficiente de perdas $-\mathrm{K} \%$ ) baseado no deságio comerci-

1. Eng ${ }^{\circ}$. Agrônomo - Estação Experimental de Itambé, Empresa IPA, Município de Itambé, Pernambuco, Brasil.

2. Dep. de Biologia - Universidade Federal Rural de Pernambuco - UFRPE. Rua D. Manoel de Medeiros, s/n, Dois Irmãos, 52171-900. Recife, PE, Brasil. 
al. Os resultados indicaram o sistema de manejo do cultivo da batatadoce (MCBd) como altamente eficiente, com 100\% de controle dos danos, nas colheitas aos 4 e 5 meses, quando, quando comparado ao $S C A g$, com incremento na produtividade ( $t / h a)$ e redução do coeficiente de perda (deságio comercial). A adubação orgânica com esterco bovino e a cobertura morta com palha seca de cana-de-açúcar não controlaram os danos da broca-da-raiz.

Palavras-chave: Batata-doce, broca-da-raiz, sistema de manejo do cultivo, sistema do agricultor, danos, perdas, produtividade.

\section{ABSTRACT}

\section{CULTIVATION MANAGEMENT OF SWEET POTATO TO CONTROL THE SWEET POTATO WEEVIL ON FARMER PROPERTIES}

The work was carried out in a private farm (Fazenda Rio Gramame), municipality of Pedras de Fogo, Paraíba, Brazil, on francareno-loamy soil, flat and without artificial irrigation (with rains only). It had in view the evaluation of the effectiveness of the system of management of the cultivation of sweet-potato (MCBd) as compared to the usual farmer method (SCAg), in the control of damage and losses caused by the sweet-potato weevil, Euscepes postfasciatus (Fairmaire), in two crop periods (4 and 5 months), at farmer level. Six randomized blocks with a factorial design of 5 managements $\times 2$ times of harvest ( 4 or 5 months) were used. The MCBd system requires immersion for 20 minutes of plantation foliage in welding insectice obtained with Clorpirifós (Lorsban $45 \mathrm{CE}$ ) at the level of $0,1 \%$ of commercial product. There was use of points of foliages and technique of the "lace" (bending at the base), to $15 \mathrm{~cm}$ of depth, in the automated system of "leirão", with chemical fertilization. In MCBd mulch with sugar cane straw was used, as well as cattle manure (AOEG). The control was farmer's conventional system (SCAg). Plantation foliages came from 
areas infested by the weevil. Damage (\%d) of the roots, yield ( $\mathrm{t} / \mathrm{ha})$ and coefficient of losses $(\mathrm{K} \%)$ and disagio of price calculated based on commercial production. Results show that the MCBd system of cultivation of the sweet-potato is highly efficient, with $100 \%$ control of damages, both for 4 and 5 months crops, with increase of productivity ( $t / \mathrm{ha}$ ) and reduction of disagio of commercial yield. Organic manure and dry straw of sugar-cane did not control the damages of sweet-potato weevil.

Key words: sweet-potato, sweet-potato weevil, system of management of cultivation, farmer's system, damages, losses, productivity.

\section{INTRODUÇÃO}

A batata-doce, Ipomoea batatas (L.) Lam. é uma dicotiledônea da Família Convolvulaceae, possivelmente originária da América Tropical, de onde foi levada para a Europa, espalhando-se, dai, para outros continentes. Mas há uma outra indicação, de que tenha origem asiática ou africana (Edmond \& Ammerman, 1971; Folquer, 1978; Peixoto \& Miranda, 1984; Barrera, 1986).

Devido à sua rusticidade, fácil cultivo, ciclo vegetativo curto e grande capacidade de adaptação às diferentes condições edafoclimáticas, acha-se muito difundida nas diferentes regiões tropicais e subtropicais do mundo, incluindo-se as mais diversas áreas fitogeográficas do Brasil (Camargo \& Noronha, 1963; Montaldo, 1966; Mafra, 1979; Raman \& Alleybe, 1991). A batata-doce é um alimento essencialmente energético e de alto valor nutritivo, hortaliça de raízes tuberosas, utilizada no consumo diário da população, especialmente a de baixa renda. É supridora contra avitaminoses, além de importante fonte de matéria prima para a indústria de doce, amido, farinha, corantes e para a alimentação de quadúpedes e aves (Edmond \& Ammerman, 1971; Peixoto \& Miranda, 1984; Folquer, 1978; Mafra, 1979). A produção no Brasil em 1995 foi de $619.186 \mathrm{t}$ para uma área colhida de 55.946 ha e rendimento médio de $11,0 \mathrm{t} / \mathrm{ha}$. Neste contexto, Pernambuco aparece em $6^{0}$ lugar (ANUÁRIO 
ESTATÍSTICO DO BRASIL, 1997). De acordo com a PRODUÇÃO AGRÍCOLA MUNICIPAL - PERNAMBUCO (1997), o Estado produziu 32.704 toneladas de batata-doce, para uma área colhida de 3.421 ha, com rendimento médio de $9,5 \mathrm{t} / \mathrm{ha}$, destacando-se as mesoregiões Agreste e Mata, com $87,4 \%$ do total produzido. O município de Itambé, Mata Norte, aparece com 3.840 t, ou seja, com 13,5\% em relação à mesoregião, mas, com rendimento médio de $12 \mathrm{t} / \mathrm{ha}$.

Diversos fatores limitam a produção e o desenvolvimento da cultura em Pernambuco, destacando-se a incidência da broca-da-raiz, $E$. postfasciatus, que ataca a parte aérea (rama) e, principalmente as raízes tuberosas, no campo e em condições de armazenamento. Sua importância como praga-chave da batata-doce resulta do fato de que toda raiz tuberosa atacada, mesmo com danos mínimos, torna-se comercialmente inaceitável, devido às alterações organolépticas que apresentam (Satô et al., 1982), havendo registros de danos no campo de 80 a 85,5\% (Vanetti, 1961; Monteiro et al., 1972; Suenaga et al., 1987). Adultos e larvas alojam-se e danificam as ramas e principalmente as raízes tuberosas, formando galerias, irregulares e descontínuas, superficiais ou profundas, com destruição total da raiz, podendo incidir sobre a cultura em qualquer fase do ciclo de desenvolvimento, e serem transportadas por ramas-sementes (Sherman \& Tamashiro, 1954; Novo, 1983; Chalfant et al., 1990; Raman \& Alleybe, 1991; Braga, 1993; Maranhão, 1994). Diversos autores têm proposto o uso do Manejo Integrado de Pragas (MIP) visando o controle de E. postfasciatus em campo (França et al., 1984; Talekar, 1989; Chalfant et al., 1990; Raman \& Alleyne, 1991; Jansson et al., 1991; EMBRAPA/CNPH, 1995).

O presente trabalho teve como objetivos avaliar a eficácia do sistema de manejo do cultivo de batata-doce quando comparado com o do agricultor, no controle dos danos e perdas (deságio comercial) causados pela broca-da-raiz, E. postfasciatus em dois períodos de colheitas (4 e 5 meses); a nível do produtor. 


\section{MATERIAL E MÉTODOS}

O experimento foi desenvolvido em 1997 em condições de campo, a nível do produtor, na fazenda Rio Gramame, município de Pedras de Fogo, Paraíba, que faz divisa com o Estado de Pernambuco através do município de Itambé, Zona da Mata Norte, a $90 \mathrm{~km}$ de Recife. O local de implantação do experimento fez parte de área reservada de cana-de-açúcar para plantio de culturas de ciclo anual, cultivada anteriormente com batata-doce. Apresenta característica estrutural uniforme, tanto de solo (franco-areno-argiloso) como de relevo (plano), com precipitação média anual de $1200 \mathrm{~mm}$ (da Fazenda e do município), distribuídos no inverno ( 70 a $80 \%$ ) e no verão ( 20 a $30 \%$ ). Usaram-se seis blocos casualizados, com tratamentos 5 manejos x 2 épocas de colheita (aos 4 ou 5 meses de plantio (Tabela 1). Cada parcela foi constituída de 10 fileiras ou leirões, com $10,0 \mathrm{~m}$ de comprimento e 33 covas por fileira, no espaçamento de $1,0 \mathrm{~m} \times 0,30$ $\mathrm{m}$, com área total de $100 \mathrm{~m}^{2}(10,0 \mathrm{~m} \mathrm{X} \mathrm{10,0} \mathrm{m).} \mathrm{A} \mathrm{área} \mathrm{útil} \mathrm{de} \mathrm{cada}$ parcela compreendeu as 4 fileiras centrais $\left(40,0 \mathrm{~m}^{2}\right)$

Tabela 1. Tratamentos utilizados no Manejo do Cultivo da Batata-doce. Pedra de Fogo, 1997.

\begin{tabular}{cl}
\hline Tratamentos & \multicolumn{1}{c}{ Especificações } \\
\hline T1a & $\begin{array}{l}\text { Manejo do cultivo da batata-doce (MCBd) + Cobertura morta } \\
\text { com palha seca de cana-de-açúcar (PSCA) } \\
\text { T1b }\end{array}$ MCBd + PSCA \\
T2a & MCBd + Adubação orgânica com Esterco bovino (AOEG). \\
T2b & MCBd + AOEG. \\
T3a & MCBd + PSCA + AEOG \\
T3b & MCBd + PSCA + AOEG \\
T4a & MCBd \\
T4b & MCBd \\
T5a & Sistema Convencional do Agricultor (SCAg) \\
T5b & SCAg \\
\hline
\end{tabular}

$\mathrm{a}$ - Colheita aos 4 meses do plantio, $\mathrm{b}$ - Colheita aos 5 meses do plantio. 


\section{Especificações sobre o manejo do cultivo da batata-doce (MCBd)}

As ramas para plantio foram obtidas na propriedade, oriundas de área infestada pela broca-da-raiz. Foram utilizadas ramas do meio para o ápice das plantas, desprezando-se a base até mais ou menos $30 \mathrm{~cm}$ do solo (ponta de ramas). $O$ tamanho das ramas variou de 35 a $40 \mathrm{~cm}$ de comprimento, sendo utilizado o cultivar Batateira (comum na região).

O sistema de plantio foi em leirão ou camalhão com preparo do solo mecanizado (aração, gradagem e sulcagem) e acabamento manual com enxada. Os leirões apresentavam $50 \mathrm{~cm}$ de altura e de 35 a $40 \mathrm{~cm}$ de largura, no espaçamento de $1,0 \mathrm{~m} \times 0,30 \mathrm{~m}$, num total de 330 covas.

No plantio das ramas-sementes, utilizou-se a técnica do laço ou trança (Mafra, 1978), com 2 ramas por cova, introduzindo-se, manualmente, $2 / 3$ das ramas no solo e $1 / 3$ permanecendo fora, numa profundidade de $15 \mathrm{~cm}$.

Em todos os tratamentos de manejo do cultivo de batata-doce (MCBd), as ramas foram imersas, por 20 minutos, em calda inseticida à base de Clorpirifós (Lorsban $45 \mathrm{CE}$ ) a $0,1 \%$ do produto comercial (100 $\mathrm{mL} / 100 \mathrm{~L}$ de água). Após o tratamento, as ramas permaneceram em repouso (à sombra) por 24 horas.

Todos os tratamentos do MCBd receberam adubação química de acordo com a análise de solo e orientação técnica (Fósforo, 1/3 do nitrogênio e $1 / 2$ de potássio, aplicados por ocasião do plantio no sulco do leirão e cobertura; e coberto por terra; o restante do nitrogênio e potássio, foi aplicado 40 dias após o plantio).

Os tratamentos $\mathrm{T} 1$ e $\mathrm{T} 3$ compreenderam cobertura morta à base de palha seca de cana-de-açúcar (PSCA), disponível na região, colocada sobre os leirões, ao lado das covas, após o pegamento das plantas.

Tratos culturais normais, com uma limpa manual, e amontoa após 45 dias do plantio, sem aplicação fitossanitária.

A contagem do stand de colheita foi realizada na área útil de cada parcela, sendo a colheita feita aos 4 ou 5 meses do plantio. 


\section{Especificações sobre o sistema convencional do agricultor (SCAg)}

O SCAg seguiu as normas de cultivo da batata-doce das propriedades familiares do município de Pedras de Fogo e adjacências; foi efetuado pelos próprios agricultores, utilizados na mão-de-obra de serviço.

O sistema de plantio foi em leirão, com o preparo do solo mecanizado, semelhante ao MCBd. O espaçamento entre os leirões foi de 1,0 m e entre covas variou de $0,20 \mathrm{~m}$ a $0,35 \mathrm{~m}$.

As ramas-sementes foram oriundas de áreas infestadas pela broca-da-raiz, utilizando-se ramas a partir da superfície do solo, da cultivar Batateira. No plantio das ramas-sementes não usou-se a técnica do "laço ou trança", introduzindo-se no solo manualmente, 2 ramas por cova, com 30 a $40 \mathrm{~cm}$ de comprimento a 10 ou $15 \mathrm{~cm}$ de profundidade (irregular).

Neste sistema não houve tratamento prévio das ramas por imersão em calda inseticida. Nos tratos culturais, ocorreu uma limpa manual aos 45 dias do plantio, sem adubação química e orgânica.

$\mathrm{O}$ tratamento SCAg constou de uma parcela experimental (semelhante ao MCBd) com 6 repetições e colheita aos 4 e 5 meses do plantio.

Efetuou-se contagem do stand de colheita na área útil de cada parcela (4 fileiras centrais), bem como a colheita.

\section{Análise estatística, obtenção dos dados de produção e medição dos} danos e perdas

O esquema de análise foi em blocos casualizados, com arranjo fatorial de 2 × 5 (Tabela 1).

As médias foram comparadas pelo teste de Tukey, ao nível de $5 \%$ de probabilidade.

Os dados de produção foram obtidos após a contagem do stand, efetuando-se a colheita, ensacamento e pesagem da produção por área útil de cada parcela. Para medição dos danos de broca-da-raiz, por ocasião da pesagem da produção por parcela, obteve-se uma amostra aleatória de $10 \mathrm{~kg}$ de batata, na qual se fez a contagem do total por amostra, com análise cuidadosa e separação das danificadas e não-danificadas, e 
cálculo do porcentual de danos (\%d). O processo de avaliação das batatas constou de um minucioso exame individual em todas as amostras. Com o auxílio de um estilete ou faca, efetuou-se raspagem da epiderme e cortes longitudinais e transversais para identificação de danos (galerias, túneis...), presença de população de $E$. postfasciatus (larvas e pupas) e atentando-se para a ocorrência do odor característico do "tungue ou tunga".

A medição das perdas foi efetuada na operação de pós-colheita, considerando-se os danos (\%d) obtidos de cada amostra de $10 \mathrm{~kg}$ por parcela. Neste caso, as batatas danificadas eram descartadas e consideradas não-comerciais. $\mathrm{O}$ deságio comercial foi calculado de acordo com o dano correspondente (\% dano x produção da parcela, em t/ha). As perdas em redução qualitativa da produção, provocadas pelo deságio comercial, foram calculadas pelo coeficiente de perda $\mathrm{K}$, utilizando-se a seguinte fórmula: $\mathrm{K}=(\mathrm{a}-\mathrm{b}) / \mathrm{a} \times 100$, onde: $\mathrm{a}=$ produção sem deságio comercial; $\mathrm{b}=$ produção com deságio.

\section{RESULTADOS E DISCUSSÃO}

Os tratamentos do manejo do cultivo de batata-doce (MCBd), que receberam, adicionalmente, imersão prévia das ramas de plantio na calda inseticida de Clorpirifós (Lorsban $45 \mathrm{CE}$ ) a $0,01 \%$ do produto comercial, durante 20 minutos e outras técnicas, obtiveram controle e proteção total dos danos na colheita aos 4 meses e reduzidos percentuais de danos $(0,0$ a $2,5 \%$ ) aos 5 meses, quando comparados ao tratamento do sistema convencional do agricultor (SCAg) o qual apresentou altos índices de danos, que variaram, em média, de 58,34 a $73,39 \%$, nas colheitas aos 4 e 5 meses do plantio, respectivamente (Tabela 2).

Ainda de acordo com a Tabela 2 e Figura 1 , o único tratamento que não apresentou danos da broca da raiz, na colheita do $5^{\circ}$ mês, foi o sistema de MCBd, que não recebeu adubação orgânica com esterco bovino (AOEG) nem cobertura morta com palha seca de cana-de-açúcar (PSCA). Estes resultados concordam com os de Braga (1993), que também testou outros tipos de cobertura morta. 
Tabela 2. Porcentual médio de dano $(\% \mathrm{~d})$ de raízes tuberosas de batata-doce, danificadas pela broca-da-raiz, em 2 épocas de colheitas. Pedras de Fogo, 1997.

\begin{tabular}{lcc}
\hline \multirow{2}{*}{ Tratamentos } & \multicolumn{2}{c}{ Épocas de colheitas } \\
\cline { 2 - 3 } & $\mathbf{4}$ meses & $\mathbf{5}$ meses \\
\hline T1 - MCBd + PSCA & $0,00 \%$ & $0,83 \%$ \\
T2 - MCBd + AOEG & $0,00 \%$ & $2,50 \%$ \\
T3 - MCBd + PSCA + AOEG & $0,00 \%$ & $0,83 \%$ \\
T4 - MCBd & $0,00 \%$ & $0,00 \%$ \\
T5 - SCAg & $58,34 \%$ & $73,39 \%$ \\
\hline
\end{tabular}

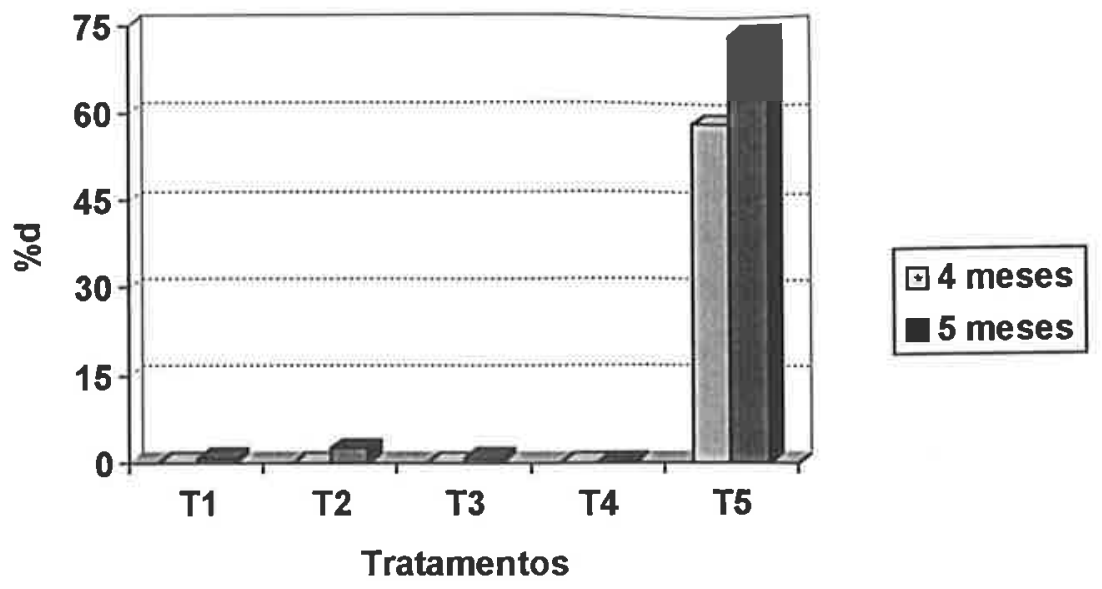

Figura 1. Diferenças entre os porcentuais de dano da broca-da-raiz, $E$. postfasciatus, em diferentes tratamentos e épocas de colheita, aos 4 e 5 meses. Pedras de Fogo, 1997.

A Tabela 3 mostra a produtividade média de batata-doce nos diferentes tratamentos e colheitas.

Quando se compararam as médias gerais dos tratamentos, resultante do somatório das duas colheitas, e os coeficientes de perdas (K\%) (Tabela 4), verificou-se aumento de produtividade nos tratamentos de manejo do cultivo da batata-doce (MCBd) com adubação orgânica com 
Tabela 3. Média de produtividade de raízes tuberosas ( $t / h a)^{*}$ de batatadoce cv. Batateira, em relação a diferentes técnicas de cultivo, com colheita aos 4 e 5 meses do plantio. Pedras de Fogo, 1997.

\begin{tabular}{lc}
\hline \multicolumn{1}{c}{ Tratamentos } & Produtividade (t/ha) \\
\hline T1 $a^{\star \star}-$ MCBd + PSCA & 18,80 \\
T1b ${ }^{\star \star}$ - MCBd + PSCA & 22,52 \\
T2a - MCBd + AOEG & 26,07 \\
T2b - MCBd + AOEG & 28,60 \\
T3a - MCBd + PSCA + AEOG & 25,86 \\
T3b - MCBd + PSCA + AEOG & 27,79 \\
T4a - MCBd & 23,09 \\
T4b - MCBd & 25,43 \\
T5a - SCAg & 10,07 \\
T5b - SCAg & 13,33 \\
\hline
\end{tabular}

Colheita aos 4 meses (a) e aos 5 meses do plantio (b).

esterco bovino (AOEG) em relação aos demais tratamentos, havendo índices de que a palha seca da cana-de-açúcar (PSCA) quando adicionada ao MCBd reduziu a produtividade. Todos os tratamentos de MCBd diferiram significativamente do $\mathrm{SCAg}$, com um incremento de produtividade máximo de $85,5 \%$ e mínimo de $81,03 \%$, com deságio.

Tabela 4. Médias* de produtividade (t/ha) dos tratamentos, com e sem deságio, e o coeficiente de perda (\%K). Pedras de Fogo, 1997.

\begin{tabular}{lccc}
\hline \multirow{2}{*}{ Tratamentos } & \multicolumn{2}{c}{ Médias (tha) ${ }^{\hbar \star}$} & \multirow{2}{*}{$\mathrm{K} \%$} \\
\cline { 2 - 3 } & Com deságio & Sem deságio & \\
\hline T1 - MCBd + PSCA & $26,98 \mathrm{a}$ & $27,33 \mathrm{a}$ & 1,28 \\
T2 - MCBd + AOEG & $26,72 \mathrm{a}$ & $26,83 \mathrm{a}$ & 0,41 \\
T3 - MCBd + PSCA + AOEG & $24,26 \mathrm{~b}$ & $24,26 \mathrm{~b}$ & 0,00 \\
T4 - MCBd & $20,56 \mathrm{c}$ & $20,66 \mathrm{c}$ & 0,48 \\
T5 - SCAg & $3,90 \mathrm{~d}$ & $11,70 \mathrm{~d}$ & 66,67 \\
\hline$\triangle 5 \%$ & 1,689 & 1,828 & - \\
CV & $6,79 \%$ & $7,95 \%$ & - \\
\hline
\end{tabular}

* Médias obtidas das duas colheitas de cada tratamento.

** Nas colunas, médias seguidas de mesma letra não diferem significativamente entre si, pelo teste de Tukey, a $5 \%$ de probabilidade. 
Ainda na Tabela 4, analisando o coeficiente de perda do tratamento $\mathrm{SCAg}=66,67 \%$ com o do $\mathrm{MCBd}=0,0 \%$ e os demais tratamentos, em relação ao deságio comercial causado pelos danos da broca-daraiz, E. postfasciatus, comprova-se a maior eficiência do sistema de manejo da batata-doce (MCBd), sem a adição de AOEG e da PSCA, mesmo porque estas variáveis aumentaram os custos do manejo e provocaram perdas $(\mathrm{K} \%)$.

Os dados das Tabelas 5 e 6 , mostram os efeitos de épocas de colheitas $\left(4^{\circ}\right.$ e $5^{\circ}$ mês $)$ dos tratamentos com deságio e sem deságio, sobre a produtividade e o coeficiente de perda $(\mathrm{K} \%)$. Com relação a produtividade, os tratamentos do sistema de manejo do cultivo da batata-doce (MCBd) não diferiram entre si, com exceção do $\mathrm{MCBd}+\mathrm{PSCA}$, com deságio, que foi o menos produtivo. No $\mathrm{SCAg}$, com deságio, não houve diferença significativa nas colheitas no $4^{\circ}$ e $5^{\circ}$ mês (Tabela 5).

Tabela 5. Efeito das épocas de colheitas na cultura da babata-doce, sobre os tratamentos com e sem deságio. Pedras de Fogo, 1997.

\begin{tabular}{|c|c|c|c|c|c|c|c|c|c|c|}
\hline \multirow{3}{*}{$\begin{array}{l}\text { Épocas } \\
\text { de } \\
\text { colheita }\end{array}$} & \multicolumn{10}{|c|}{ Produtividade (t/ha) ${ }^{*}$} \\
\hline & \multicolumn{5}{|c|}{ Com deságio } & \multicolumn{5}{|c|}{ Sem deságio } \\
\hline & $T 1^{\star \star}$ & T2 & T3 & T4 & T5 & T1 & $\mathrm{T} 2$ & T3 & T4 & T5 \\
\hline $5^{\circ}$ mês & $22,27 a$ & $27,90 a$ & $27,59 a$ & 25,43 a & $3,61 \mathrm{a}$ & 22,52 a & $28,60 \mathrm{a}$ & $27,78 a$ & $25,43 a$ & $13,33 a$ \\
\hline $4^{\circ}$ mês & $18,80 \mathrm{~b}$ & $26,00 \mathrm{a}$ & $25,86 \mathrm{a}$ & $23,09 a$ & $4,18 \mathrm{a}$ & $18,80 \mathrm{~b}$ & $26,00 \mathrm{a}$ & 25,86 a & $23,09 \mathrm{~b}$ & $10,07 \mathrm{~b}$ \\
\hline $\begin{array}{l}\Delta 5 \% \\
C V\end{array}$ & $\begin{array}{r}2,585 \\
7,95 \%\end{array}$ & & & & & $\begin{array}{l}2,334 \\
679 \%\end{array}$ & & & & \\
\hline
\end{tabular}

* Nas colunas, médias seguidas de mesma letra não diferem entre si, pelo teste de Tukey, a $5 \%$ de probabilidade.

** $\mathrm{T} 1=\mathrm{MCBd}+\mathrm{PSCA}, \mathrm{T} 2=\mathrm{MCBd}+\mathrm{AOEG}, \mathrm{T} 3=\mathrm{MCBd}+\mathrm{PSCA}+\mathrm{AOEG}, \mathrm{T} 4=$ MCBd e T5 $=$ SCAg.

Quanto ao coeficiente de perdas (K\%), o MCBd foi o único que se manteve estável, com $0,0 \%$ de perdas, do $4^{\circ}$ para o $5^{\circ}$ mês de colheita, enquanto o SCAg apresentou um aumento de perdas de $14,43 \%$, respectivamente. Deduz-se então que com o deságio comercial, o sistema de MCBd foi o mais eficiente em prudutividade e coeficiente de perdas (K\%) em relação às variáveis adicionais (adubação orgânica - AOEG e palha seca de cana-de-açúcar) e, sobretudo, em relação ao SCAg (Tabela 6). 
Tabela 6. Médias de produtividade (t/ha)* de cada tratamento, com e sem deságio em relação à duas épocas de colheitas e o coeficiente de perdas (K\%). Pedras de Fogo, 1997.

\begin{tabular}{|c|c|c|c|c|c|c|}
\hline \multirow{3}{*}{$\begin{array}{c}\text { Trata- } \\
\text { mentos* }\end{array}$} & \multicolumn{4}{|c|}{$\begin{array}{l}\text { Produtividade (t/ha) } \\
\end{array}$} & \multirow{2}{*}{\multicolumn{2}{|c|}{$\mathbf{K} \%$}} \\
\hline & \multicolumn{2}{|c|}{ Com deságio } & \multicolumn{2}{|c|}{ Sem deságio } & & \\
\hline & $4^{\circ}$ mês & $5^{\circ}$ mês & $4^{\circ}$ mês & $5^{\circ}$ mês & $4^{\circ}$ mês & $5^{\circ}$ mês \\
\hline T1 & $26,00 \mathrm{a}$ & $27,90 \mathrm{a}$ & $26,00 \mathrm{a}$ & $28,60 a$ & 0,00 & 0,57 \\
\hline $\mathrm{T} 2$ & $25,86 a$ & $27,59 \mathrm{a}$ & 25,86 a & $27,78 \mathrm{ab}$ & 0,00 & 0,68 \\
\hline T3 & $23,09 \mathrm{~b}$ & 25,43 a & $23,09 \mathrm{~b}$ & $25,43 \mathrm{~b}$ & 0,00 & 0,00 \\
\hline T4 & $18,80 \mathrm{c}$ & $22,27 \mathrm{~b}$ & $18,80 \mathrm{c}$ & $22,52 \mathrm{c}$ & 0,00 & 1,11 \\
\hline T5 & $4,18 \mathrm{~d}$ & $3,61 \mathrm{c}$ & $10,07 \mathrm{~d}$ & $13,33 \mathrm{~d}$ & 58,49 & 72.92 \\
\hline$\Delta 5 \%$ & 2,389 & & 2,525 & & & \\
\hline CV & $6,79 \%$ & & $7,95 \%$ & & & \\
\hline
\end{tabular}

* Nas colunas, médias seguidas de mesma letra não diferem entre si, pelo teste de Tukey, a $5 \%$ de probabilidade.

** $\mathrm{T} 1=\mathrm{MCBd}+\mathrm{PSCA}, \mathrm{T} 2=\mathrm{MCBd}+\mathrm{AOEG}, \mathrm{T} 3=\mathrm{MCBd}+\mathrm{PSCA}+\mathrm{AOEG}, \mathrm{T} 4=$ MCBd e T5 $=$ SCAg.

\section{CONCLUSÕES}

- O sistema de manejo do cultivo de batata-doce (MCBd) é altamente eficiente, alcança $100 \%$ de controle em relação aos danos da broca-da-raiz, Euscepes postfasciatus, nas colheitas aos 4 e 5 meses, em relação ao sistema convencional do agricultor (SCAg), que apresentou 58,34 e 73,39\% de raízes tuberosas danificadas, respectivamente.

- A adubação orgânica com esterco bovino (AOEG) e cobertura morta com palha seca de cana-de-açúcar (PSCA) foram ineficientes no controle dos danos da broca-da-raiz.

- O sistema de manejo do cultivo da batata-doce (MCBd) aumenta a produtividade e reduz o coeficiente de perdas $(\% \mathrm{~K})$ em relação ao deságio comercial, quando comparado com o sistema convencional do agricultor ( $\mathrm{SCAg}$ ), independentemente do mês de colheita. 


\section{REFERÊNCIAS BIBLIOGRÁFICAS}

ANUÁRIO ESTATÍSTICO DO BRASIL-IBGE, 1997. Rio de Janeiro, v.57, p.3-41.

BARRERA, P., 1986. Batata-Doce. Icone. São Paulo, 91p.

BRAGA, E.C., 1993. Resistência de Cultivares de Batata-Doce (Ipomoea batatas (L.) Lam.) e a Eficiência da Cobertura Morta no Controle da Broca-da-Raiz, Euscepes postfasciatus (Fairmaire, 1849) (Coleoptera,

Cucurlionidae) em Pernambuco. Recife, 59p. Dissertação (Mestrado em Fitossanidade). Departamento de Agronomia /UFRPE: CAMARGO, H.P.; NORMANHA, E.S., 1963. Instruções Para o Cultivo da Batata-Doce. $2^{a}$ ed. Campinas:IAC. Boletim n ${ }^{\circ} 43,25 p$. CHALFAN, R.B.; JANSSON, R.K.; SEAL, D.R.; CHALK, Y.M., 1990. Ecology and Management of Sweet Potato Insects. Ann. Rev. of Entomol., 35:157-180.

EDMOND, J.B.; AMMERMAN, G.R., 1971. Sweet Potato: Production,

Processing, Marketing. Connecticut, The AVI Publishing Company, Inc., 334p.

FOLQUER, F., 1978. La Batata (Camote): Estudo de la Planta y su

Producción Comercial. Buenos Aires, Argentina, 144p.

FRANÇA, F.H.; MIRANDA, J.E.C.; BARBOSA, S.; CORDEIRO, C.M.T.; COELHO, M.C.F. Manejo Integrado de Pragas da BatataDoce. In: CONGRESSO BRASILEIRO DE OLERICULTURA, 24. e REUNIÃO LATINO-AMERICANA DE OLERICULTURA, 1, Brasília: Sociedade de Olericultura do Brasil, Resumos, p.137. EMBRAPA-CNPH, 1995. Cultivo de Batata-Doce. Instruções Técnicas $7,18 \mathrm{p}$.

JANSSON, R.K.; RAMAN, K.V.; MALAMUD, O.S., 1991. Sweet Potato Pest Management: Future Outlook. In: Sweet Potato Pest Management. A global Perspective. Westview Press, Colorado, USA, p.430-437. MAFRA, R.C., 1979. Cultura da Batata-Doce. Recife, UFRPE, 27p. MARANHÃO, L.M.A.C., 1994. Estudo da Broca da Raiz da BatataDoce, Euscepes postfasciatus (Fairmaire, 1849) (Coleoptera, Cucurlionidae), Ocorrência de Parasitóides e Controle Microbiano 
em Pernambuco. Recife, 150p. Dissertação (Mestrado em Fitossanidade). Departamento de Agronomia/UFRPE: MONTALDO, A., 1966. Manual de Cultivo de la Batata (Ipomea batatas Lam.).

Maracay, Universidad Central de Venezuela, Faculdad de Agronomia, 44p. MONTEIRO, D.A.; PARRA, J.R.P.; CAVALCANTE, R.D.; IGUE, T., 1977. Controle da Broca da Batata-Doce Euscepes postfasciatus (Fairmaire, 1849) (Coleoptera, Cucurlionidae), com Inseticidas Modernos. O Biológico, 38(7):204-206.

NOVO, R.J., 1983. Alternativas de Controle de Megastes spp. (Lepidoptera, Pyralidae) e Euscepes postfasciatus (Fairmaire, 1849) (Coleoptera, Cucurlionidae) em Batata-Doce (Ipomoea batatas (L.) Lam.) no Estado de Pernambuco. Recife, 113p. Dissertação (Mestrado em Fitossanidade). Departamento de Agronomia/UFRPE: PEIXOTO, N.; MIRANDA, J.E.C., 1984. O Cultivo da Batata-Doce em Goiás. EMGOPA, Goiania, Circular Técnica n.7, 24p.

PRODUÇÃO AGRÍCOLA MUNICIPAL - PERNAMBUCO, 1997. IBGE, Rio de Janeiro, p.10-13.

RAMAN, K.V.; ALLEYNE, E.H. Biology and Managment of West Indian Sweet Potato Weevil, Euscepes postfasciatus (Fairmaire, 1849). In: Sweet Potato Pest Management. A global Perspective. Westview Press, Colorado, USA, p.263-281.

SATÔ, K; URITANI, I.; SAITO, T.; HONDA, H., 1978. Factor Causing Terpene Induction in Sweet Potato Roots Extracted from West Indian Sweet Potato Weevil, Euscepes postfasciatus Fairmaire (Coleoptera, Curculionidae). Applied of Entomol. Zoology, 13(3):227-228.

TALEKAR, C.S., 1982. Effects of a Sweet Potato Weevil (Coleoptera, Curculionidae) Infestation on Sweet Potato Root Yields. Journal of Econ. Ecol., 75:1042-1044.

TALEKAR, C.S., 1989. Development and Testing of na Integrated Pest Management Technique to Control of Sweet Potato Weevil. In: Improvement of Sweet Potato (Ipomea batatas) in Asia. p.117-126. International Potato Center, Lima, Perú.

VANETTI, F., 1961. Efeitos da Aplicação de Inseticidas no Combate a Broca da Batata-Doce. FIR, 3(11):46-48. 